\title{
Online Mingling: Supporting Ad Hoc, Private Conversations at Virtual Conferences
}

\author{
Jaeyoon Song \\ jaeyoons@mit.edu \\ MIT Center for Collective Intelligence \\ Cambridge, Massachusetts, USA
}

\author{
Christoph Riedl \\ c.riedl@northeastern.edu \\ Northeastern University \& MIT \\ Center for Collective Intelligence \\ Boston, Massachusetts, USA
}

\author{
Thomas W. Malone \\ malone@mit.edu \\ MIT Center for Collective Intelligence \\ Cambridge, Massachusetts, USA
}

\begin{abstract}
Even though today's videoconferencing systems are often very useful, these systems do not provide support for one of the most important aspects of in-person meetings: the ad hoc, private conversations that happen before, after, and during the breaks of scheduled events-the proverbial hallway conversations. Here we describe our design of a simple system, called Minglr, which supports this kind of interaction by facilitating the matching of conversational partners. We describe two studies of this system's use at two virtual conferences with over 450 total participants. Our results provide evidence for the usefulness of this capability, showing that, for example, $81 \%$ of people who used the system successfully thought that future virtual conferences should include a tool with similar functionality. We believe that similar functionality is likely to be widely implemented in many videoconferencing systems and to increase the feasibility and desirability of many kinds of remote work and socializing.
\end{abstract}

\section{CCS CONCEPTS}

- Collaborative and social computing $\rightarrow$ Collaborative and social computing systems and tools; Empirical studies in collaborative and social computing.

\section{KEYWORDS}

virtual conferences, social interaction, ad hoc conversations, videoconferencing, online mingling

\section{ACM Reference Format:}

Jaeyoon Song, Christoph Riedl, and Thomas W. Malone. 2021. Online Mingling: Supporting Ad Hoc, Private Conversations

at Virtual Conferences. In CHI Conference on Human Factors in Computing Systems (CHI '21), May 8-13, 2021, Yokohama, Japan. ACM, New York, NY, USA, 10 pages. https://doi.org/10.1145/3411764.3445776

\section{INTRODUCTION}

As a result of the COVID-19 pandemic in the spring of 2020, many people learned suddenly (and often somewhat involuntarily) that Zoom, Skype, Facetime, and other videoconferencing systems could be surprisingly good for many kinds of scheduled meetings. These

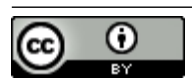

This work is licensed under a Creative Commons Attribution International 4.0 License.

CHI '21, May 8-13, 2021, Yokohama, Japan

(C) 2021 Copyright held by the owner/author(s)

ACM ISBN 978-1-4503-8096-6/21/05

https://doi.org/10.1145/3411764.3445776 tools are not always as good as meeting in-person, but they are often close, and sometimes even better.

As many people have also experienced, however, there is at least one very important thing that these tools do not do well. That is supporting the kind of ad-hoc, private conversations people often have before or after meetings, in the hallway during the breaks of a meeting, or around the office coffee machine.

As the prior research summarized below suggests, these ad hoc, random encounters can be key to creative innovations in cities, research labs, companies, and elsewhere. They can also be critical to forming social bonds and building trust in a group. In fact, we show with a survey that these ad hoc conversations are one of the things people value most about in-person conferences, and we suspect that these interactions are among the things people miss most about working from home and attending virtual conferences or other meetings.

However, most people do not realize how straightforward it can be to create videoconferencing software that supports these ad hoc interactions. In this paper, we describe one such simple system, called Minglr, and its use at two virtual conferences. We also show through the analysis of surveys, system log data, and interviews that the system was usable and that conference participants found its functionality highly useful.

We believe that, together, this system and our study of its use demonstrate both the surprising simplicity and the surprising value of supporting these ad hoc, private conversations online. We have made this system available as open source software, we expect similar functionality to be added to other videoconferencing systems, and we believe that, as these things happen, many kinds of remote work and socializing will become more common.

\section{BACKGROUND}

\subsection{The Importance of Ad Hoc Interactions}

Random interactions are critical for creativity and innovation. Such encounters may play a key role in innovation as they expose individuals to new sources of information that can lead to the generation of new knowledge [14, 16, 26, 34], and breakthrough discoveries often involve unexpected combinations of ideas [28] and new collaborations [5]. For example, research by Boudreau [5] shows that serendipitous, face-to-face encounters among medical researchers during a short 90-minute large-scale information-sharing session resulted in a $75 \%$ increase in probability of co-authoring grant applications. The benefits of random encounters are also apparent on even larger scales. For example, research shows that various measures of innovation in cities increase superlinearly with the size of a city $[3,4]$. A city that is twice as large as another one, for instance, 
has about $15 \%$ higher per capita rates of patents, R\&D employment, and other measures of innovation. A plausible explanation for this increase is the increase in opportunities for productive, random encounters that larger cities provide. In addition to the direct benefits of information exchange, these random interactions can also have more indirect positive effects by forming social bonds and building trust in a group [9].

Random encounters are also one of the key benefits and desired goals of attending an academic conference. Serendipitous or "chance" encounters at conferences and other gatherings provide individuals with non-routine opportunities for face-to-face interaction and rich knowledge sharing [15]. Researchers have significant freedom in choosing who to collaborate with [30]. However, search costs and frictions introduce significant hurdles in the formation of scientific collaborations [5] and have been blamed for lower rates of innovation, success, and reproducibility [12]. In fact, social interactions between people whose offices are more than a few yards apart are extremely rare $[2,7,13,27]$. These results all highlight the potential value of random encounters at a conference, where people from all around the world gather and communicate with one another outside of scheduled meetings.

\subsection{The Increasing Importance of Virtual Conferences}

Two global trends suggest a shift away from large in-person meetings to virtual meetings. First, due to concerns about climate change and efforts to reduce carbon impact, companies and academic institutions are attempting to reduce air travel and offset carbon emissions for unavoidable travel which increases costs [17, 20, 36]. For example, Microsoft has committed to cut operational carbon emissions by $75 \%$ by 2030 [6], and there are direct calls for the academic sector to reduce air travel [18]. Such goals are only possible with reduced air travel which is a major contributor to carbon emissions [31].

Second, global pandemics like COVID-19 introduce health risks that make large in-person gatherings impossible. These two trends are furthermore related as climate change acts as a risk multiplier that makes future pandemics more likely due to the destruction of natural animal habitats and higher temperatures [37].

Together, these two trends suggest why virtual meetings are becoming increasingly common and important both in academia and business. In the last few months, with the coronavirus pandemic, vast numbers of people have learned to use videoconferencing technologies. Tech companies like Facebook and Twitter are leading the way in making the transition to remote work brought about by the coronavirus pandemic permanent [8], and many academic conferences are now being held online [1,29].

\section{RELATED WORK}

Two main requirements are needed for random encounters to occur: (1) a way of finding people to communicate with (i.e., a matching process) and (2) a way of communicating with the people you have matched with (i.e., a communication process).

While a significant body of research has worked on facilitating social interactions at virtual meetings, the focus has been mostly on bringing the feeling of physical proximity to the communication process, and less on improving the matching process. For instance, GAZE-2 improves videoconferencing technology by transmitting eye-contact information, which is intrinsic to the collocated meeting [35]. TeleHuman uses a 3D screen to display life-size video of a person, increasing the sense of social presence [22].

There have been few studies around supporting the matching process. One notable exception is Sidebar [11], a room-to-room videoconferencing system that tracks meeting participants in realtime through image analysis of the conference video feed. It allows each participant to identify and track other participants, to look up information about them and their local work context, and to engage in peer-to-peer chat conversations. While Sidebar provides users with the awareness of each other and opportunities to connect to each other, it does not provide further information to enhance the efficiency of the matching process. For example, a Sidebar user cannot know whether someone is currently talking to another user or not.

In addition, there have been several commercial products that focus on the matching process. Donut is a matchmaking bot that automatically matches two random people from a team based on their schedules [10]. It suggests to the matched pair to go out for coffee and spend time together. Grip also automatically matches the participants at a conference using information about the participants and their stated preferences [24]. Unlike these systems, we focused on keeping humans in control of the matching process instead of fully automating it.

There are also tools like Qube, Sococo, and Online Town that simulate details of a physical space in an online environment with, for example, avatars of people moving around in the space [19, 32, 33]. We decided not to try to replicate these physical aspects of informal mingling, because we wanted to explore the degree to which abstracting away from the physical details could provide a simpler and more fulfilling user experience.

\section{PRELIMINARY STUDY}

Are ad-hoc interactions important at in-person meetings and should virtual meetings support them as well? To evaluate the relative importance of different aspects of conferences and identify potential problems with conferences that might indicate important design goals, we invited members of the CHI community to fill out a webbased survey. (See Appendix A in supplementary material for the text of the survey and the list of mailing lists and social media pages through which we solicited survey respondents.) We received 53 responses to the survey, and all respondents had experience of attending in-person conferences. The 30 respondents who said they had attended virtual conferences were also asked to report their experiences with virtual conferences.

We chose the CHI community as the population for our preliminary study because it was relatively representative of a much broader category of researchers and practitioners who attend conferences in technical and scientific areas. It was also a community that we had straightforward means to access (i.e., the mailing lists). We conducted a web-based survey because it can help gauge individual views and experiences in qualitative and quantitative ways [23]. 
(a) How important are the following parts of an in-person conference for you personally? $(\mathrm{N}=53)$

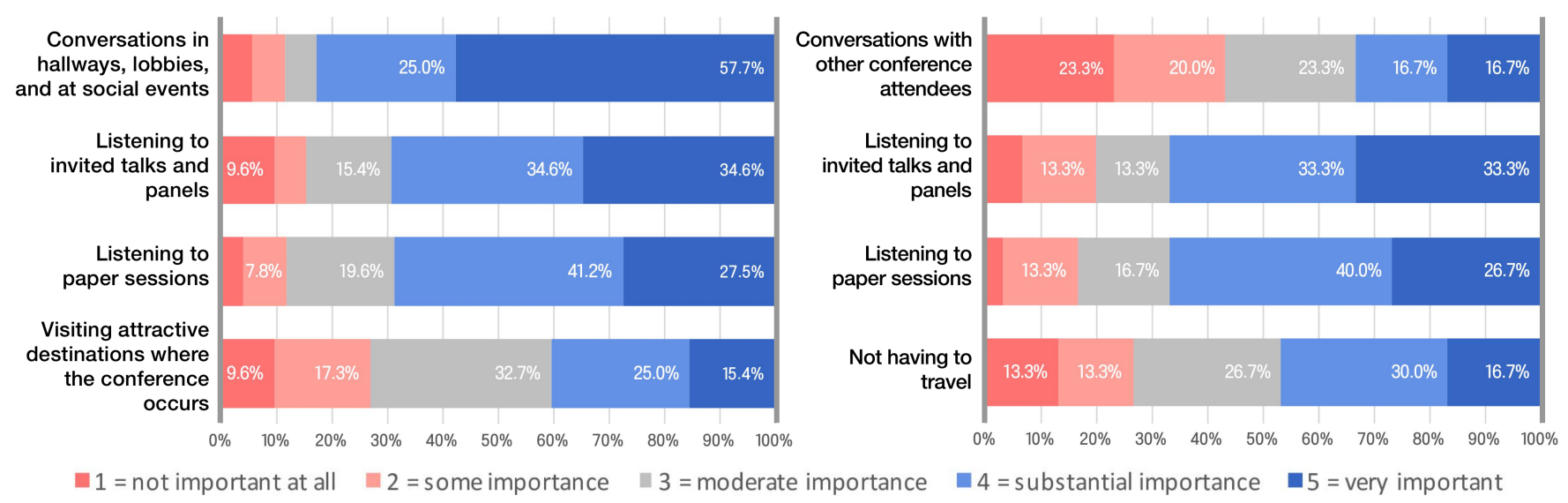

Figure 1: Ratings for value of different aspects of (a) in-person and (b) virtual conferences

\subsection{The Relative Importance of Ad-Hoc Conversations at Conferences}

According to this survey, the most important part of attending in-person conferences is the conversations in hallways, lobbies, and at social events-more important than listening to keynote or paper presentations (Figure $1(\mathrm{a}), \chi^{2}=4.68 ; p=0.03$ ). This indicates that facilitating ad hoc conversations is crucial and thus supports the importance of our overall system design goal. The survey also shows that such conversations with other attendees are not very important in virtual conferences (Figure 1(b)), presumably because they are not well supported in current virtual conferences. While the responses for other aspects of conferences are similar between in-person and virtual conferences, the average rating of the importance of conversations is significantly lower for virtual conferences than for in-person conferences $(p<0.001)$.

\subsection{Problems of Virtual Conferences}

To gain a better understanding of the challenges faced by virtual conferences, we asked the respondents to the survey what they liked least about virtual conferences. The overwhelming majority (21 out of 25) mentioned the lack of opportunities for casual conversation as the worst part of virtual conferences. One respondent said, "There is no space for informal discussion and meeting people. When the official part ends, people tend to quit, especially those figures in the field - meeting them at the conference is often a unique occasion to catch them not that busy."

Asked about what they wanted future virtual conferences to be like, half of the respondents (10 out of 19) mentioned the needs for informal interactions. Said one respondent "I wish virtual conferences could have a better channel to allow people to interact with others in a more informal way than we can currently do."

Although these results may not be representative of all conference attendees, they clearly suggest that support for ad-hoc conversations is critical and highly desired for virtual conferences. About half of respondents also mentioned not having to travel as a benefit of virtual conferences. So if the challenge of facilitating (b) How important are the following parts of a virtual conference for you personally? $(N=30)$

ad-hoc meetings at virtual conferences could be overcome, virtual conferences could provide significant advantages over in-person meetings.

\subsection{Difficulties in Socializing at In-Person Conferences}

We asked the survey respondents what challenges, if any, they have experienced when socializing at in-person conferences. Here we summarize key findings drawn from the responses.

4.3.1 Challenges for shy people and newcomers. Thirteen respondents (37\%) emphasized the challenges faced by shy people and those who don't already know others in the group. Responses suggest that many people tend to stick to the groups they came with, which makes it difficult for newcomers or shy people to mingle with the others. A respondent mentioned, "I have a hard time starting conversations with people I don't know due to mild social anxiety." This suggests the need for matching support based on profile information or even randomly.

4.3.2 Accessibility of superstars. Four people (11\%) pointed out the low accessibility of senior scholars as a difficulty. A respondent said, "big guys are always too busy and I need to wait in a long line to get a chance to talk to them." Responses also suggest that waiting in line for one person at a time can be inefficient particularly when waiting for a famous researcher or speaker. This suggests a need for the ability to queue for multiple conversation partners simultaneously to increase matching efficiency.

4.3.3 The difficulty of deciding who to talk to. Ten respondents (29\%) mentioned that it is hard to find an appropriate person to talk to at in-person conferences. A respondent pointed out "not knowing how to find who to go to meals with" as a major difficulty. This indicates the need for a structured and easy way to initiate a conversation. Responses also suggest that having too many people at the conference increases the search cost. It is easy to miss many people, and it is hard to tell who is open to being approached. One respondent mentioned that "it is hard to assess if you are important 

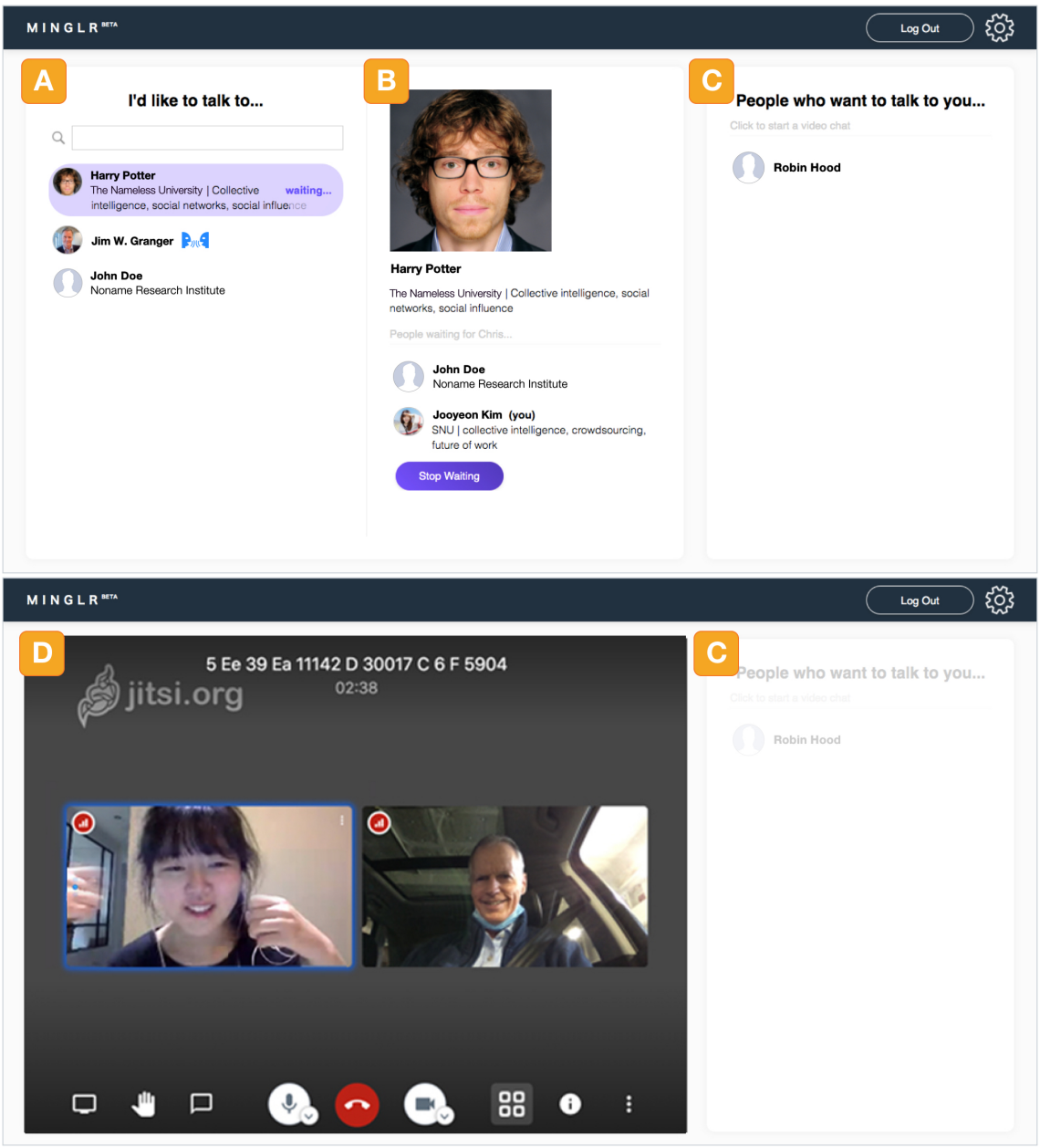

Figure 2: The Minglr interface

enough to be worth someone's time, given that everyone is there with their own ambitions and intentions."

\section{DESIGN OF THE MINGLR SYSTEM}

\subsection{Design Goals}

Our overall goal in designing the Minglr system was to support online the kind of informal conversations that happen around the edges of in-person meetings, such as the hallway conversations at in-person conferences. Stated more precisely, our first design goal is to:

- G1: Provide a very easy way for people in an online group to have private videoconferences. In a conventional videoconference, the group members need to (a) schedule a time for the meeting, (b) share a URL or other information needed to join the meeting, and (c) actually join the meeting. Our goal is to simplify this process as much as possible.

We also wanted to provide some functionality that would be better than in-person mingling in cases where that could be done easily. In particular, we wanted to take advantage of the non-physical environment to facilitate efficient matching of conversational partners in useful ways that would be difficult or impossible to do in-person. This leads to two additional design goals:

- G2: Support the process of finding potential conversational partners. In large groups or in groups where many people do not know each other, it can sometimes be difficult (especially for shy people and newcomers) to find others they want to talk to. Our second goal is to facilitate this process by, for example, making it easier for people to see information about each other and to find others with similar interests [25].

- G3: Enhance the efficiency of matching. In many meetings, some people (such as keynote speakers at a conference) are in high demand while others may be unable to find conversational partners. In in-person meetings, the process of matching conversational partners is constrained by who is nearby physically. Our third goal is to try to improve the efficiency of this process by, for example, allowing people to wait simultaneously for any number of people.

A non-goal of this project was to try to replicate the physical aspects of informal mingling, such as the effect of physical proximity on ability to hear or talk to others. In other words, we did not want 
to try to provide an online environment that simulated details of a physical space with, for example, avatars of people moving around in the space. Instead, we wanted to explore the degree to which the simplicity of abstracting away from these details could provide a more fulfilling user experience.

\subsection{System Functionality}

To achieve these goals, we implemented the following functionality, in order of typical usage.

5.2.1 Creating a profile. Users first register for the system by either using their Google or Facebook logins or by creating a new account for Minglr using their email address. As soon as they log in, they get an opportunity to edit their Minglr profile to include their affiliation and research interests (supports goal G2). In addition, if Minglr has not already imported a picture of them from their Google or Facebook login, they are able to upload a profile picture of themselves.

5.2.2 Finding conversational partners. Profiles of active users appear in the list on the left (Figure 2, part A). Each time a user clicks on a profile, information about that person and others waiting to talk to that person appears in the middle of the window (part B). Unlike in-person conferences, where you can sometimes see limited information about a person if you can get close enough to them to read their name badge, in Minglr, you can easily see not only the names and affiliations of anyone else in the group but also any other information they have entered about their interests. Minglr also provides a search function with which you can easily search for people with specific keywords in their affiliation or interests. These features support the process of searching for a conversation partner and reduce the users' difficulty of deciding who to talk to (G2).

To address the difficulties faced by shy or novice people, the list of profiles is randomized differently for each user (G2). If we had, for instance, listed the names alphabetically, then it is likely this would have given more interaction opportunities to people whose names came early in the alphabet.

5.2.3 Matching conversation partners. The system provides a very easy way for people to have private videoconferences (G1). On the left (Figure 2, part A), users can select people they want to talk to from the list of other people participating in the meeting. To increase the efficiency of matching, Minglr allows a user to wait for more than one user at a time and shows a special icon that indicates people who are already talking to someone else (G3). Still, users who want to wait for these people are free to do so. On the right side of the window (part C), users see other people who want to talk to them. And when they select one of those names, the two people are placed in a private videoconference with each other (see Figure 2).

5.2.4 Supporting private video conferences. Once a match has been made, pairs of users are placed in a private videoconference room (Figure 2, part D) where they can talk to each other as in a typical videoconference for as long or as short a time as they want. During their conversation, they can also see (part C) a continually updated list of other people who want to talk to them. And whenever either user is ready to end the conversation, they can do so by clicking the "hangup" button near the bottom of the window which returns them to the previous screen (Figure 2).

In the interests of simplicity of the system and data, we did not provide several other features that seem desirable for future extensions, including the ability to (a) have more than two people in a private videoconference, (b) automatically match users with similar interests, and (c) randomly match pairs of users who both want to be matched this way.

\subsection{Implementation}

We implemented Minglr as a JavaScript-based web app, and we used an iterative design process of prototyping and pilot studies. Minglr builds upon jitsi, an open source videconferencing system [21], and Minglr is also available as open source software (see https://github.com/CCI-MIT/minglr).

\section{DEPLOYMENT STUDY}

\subsection{Participants and Procedure}

Study 1. We first deployed the Minglr system at the ACM Collective Intelligence conference (CI 2020), a one-day academic conference held virtually on June 18, 2020. The conference lasted from 9:00 am to $4: 45 \mathrm{pm}$ EDT. All the main conference sessions happened in a Zoom meeting, with all attendees except scheduled speakers muted. A total of 275 unique individuals attended the Zoom meeting over the course of the day (average duration of attendance was $30 \mathrm{~min}$ [95\% confidence interval: 20-39]).

The first session of the conference included a series of keynote presentations, one of which included, among other topics, a description of Minglr. Then conference participants were invited to use Minglr starting in the first break of the conference, and the system remained available for their use throughout the rest of the day. To use Minglr, participants went to the Minglr website using their browsers, and they could also keep Zoom open in a separate window if they wanted to.

Study 2. The second study took place at the Microsoft New Future of Work Symposium (NFW), a virtual conference held for three days from August 3 to 5, 2020. The conference lasted from 9:00 am to 12:00 pm PDT each day. Microsoft Teams was used for all the events. Similarly, all attendees except scheduled speakers were muted.

Approximately 190 unique individuals attended the conference on the first day. At the beginning of the conference, one of the chairs announced the Minglr system in the text chat of Teams, and a plenary speaker described it in the second session of the day. Most of the participants started to use Minglr at the attendee networking session, which was from 11:10 am to 12:00 pm. As in Study 1, participants used Minglr in their browsers, and they had the option of keeping Teams available in a separate window.

\subsection{Data Collection}

To better understand how Minglr was used and how its users evaluated it, we collected three kinds of data at each conference:

(1) System usage log. During the conference, the Minglr system automatically logged all operations the users performed using the system. For example, the log includes each time a 


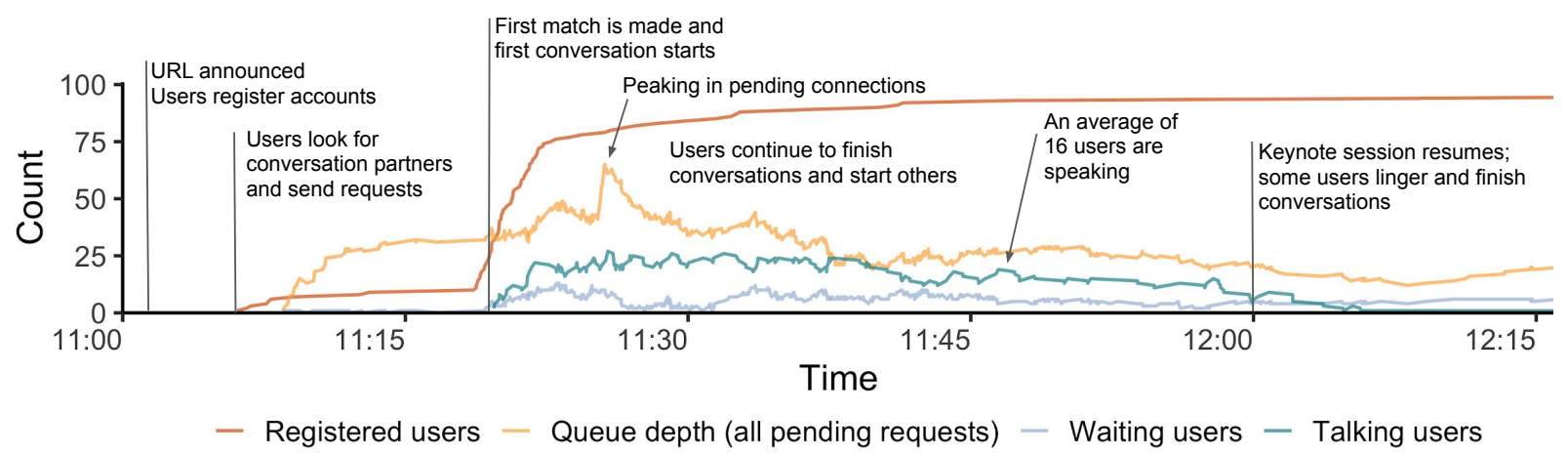

Figure 3: Timeline of Minglr usage during Study 1 (ACM CI 2020).

user requested to speak to another user, accepted a request to speak, began a conversation, or ended a conversation. The log does not include any aspect of the actual conversations users had with each other.

(2) Post-conference survey. After the conference ended, we sent a web-based survey to all conference registrants. The survey included various multiple choice and open-ended questions about the users' evaluations of various aspects of the overall conference and of the Minglr system in particular. (See Appendix B in supplementary material for the complete text of this survey.) Of the 275 attendees who participated in Study 1,71 responded to the web survey (a $26 \%$ response rate). In Study 2, 24 out of 190 attendees responded to the survey (a $13 \%$ response rate).

(3) Post-conference interviews. To complement the survey data with a qualitative understanding of the users' experience, we conducted semi-structured interviews with five survey respondents from Study 1: three professors, one student in preparation for graduate school, and one employee at a research institute. Each interview session lasted about 30 mins.

All data collection for this study (and the preliminary survey) was determined to be "Exempt" by the IRB at the MIT Committee on the Use of Humans as Experimental Subjects (project no. E-2306).

\section{RESULTS}

\subsection{System Usage Log}

We recorded usage logs for both studies. In Study 1, we announced the URL to Minglr shortly after 11:00 am, just before the first scheduled break at 11:10 am (Figure 3). The first conversation started at 11:19 am and all conversations finished by 12:05 pm when the next session of keynote presentations resumed (10 additional conversations took place during the rest of the day). Over one third of all conference participants (103 out of 275) used Minglr (i.e., they signed up and had at least one conversation).

Users placed 300 requests to speak with other users, which were matched in a total of 80 dyadic conversations, with a cumulative total of 7.5 hours of conversation time. Users had an average of 2 conversations [min 1, max 10]. Minglr facilitated an average of 8 conversations simultaneously during those times when any conversations were occurring. Conversations lasted on average 5 minutes 37 seconds [ $\min 8$ seconds; $\max 29$ minutes]. The average conversation time per user was 15 minutes. Note that all these conversations were formed on an ad hoc basis, facilitated by the Minglr matching system. None of them were scheduled or planned in advance.

Minglr was efficient at matching conference participants to conversation partners. Among all registered users, 76\% were successfully matched with at least one conversation partner (79 out of 104). To arrive at these conversations, users indeed leveraged the advantage of a virtual system and queued up on average 5.4 others to talk to. This suggests that the system design was effective in facilitating efficient matching. Among users who were part of a conversation request (either as the initiator or the invitee), 94\% (79 out of 84) were successfully matched and placed in at least one conversation. The average waiting time between placing a conversation request and being matched was only 9 seconds, plus or minus 4 seconds. Conference participants are linked in a dense network through an integrated community: 74\% of participants in Study 1, for example, are connected to each other (Figure 4). This suggests that participants had diverse conversation partners, and some of the most popular conversation partners were neither speakers or organizers of the conference.

We found similar usage pattern during the deployment in Study 2 (Figure 5). Minglr efficiently matched 103 registered users into 109 ad hoc conversations. $84 \%$ of users who sent at least one request ended up having at least one conversation, resulting in a similarly dense social network.

\subsection{Quantitative Questionnaire Results}

7.2.1 Use of Minglr. In Study 1, among the 70 respondents who answered the question "Did you use Minglr?", 29 said yes and 41 said no. Of those who did not use Minglr, the most common reason given for not using Minglr was that they wanted to take a break or do other things, which was given by $56 \%$. Other reasons chosen were: could not get it to work (12\%), were not interested in talking with others (7\%), or "other" (24\%). In Study 2, all respondents except two answered that they did use Minglr.

7.2.2 Value of Functionality like Minglr. Perhaps the most important question about Minglr is the one shown in Table 1. To assess the value of the general functionality provided by Minglr, not the specific details of our current implementation of this functionality, 


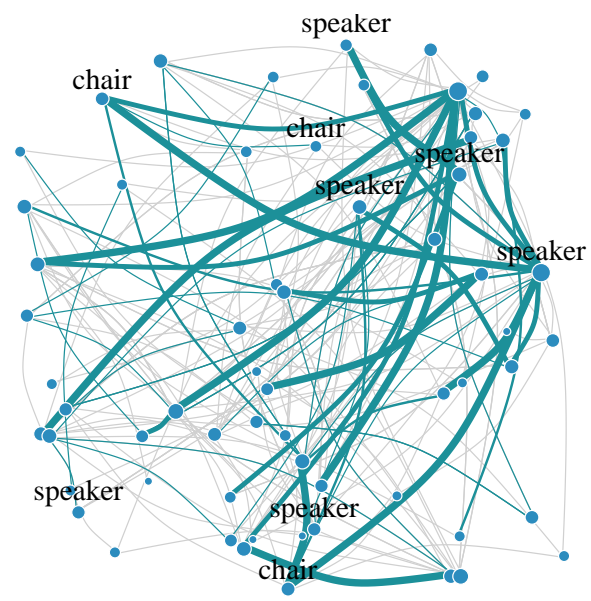

Figure 4: Communication network during the ACM CI 2020 conference. Edges shown in grey are chat requests, those in green are conversations weighted by length. Node size scaled by degree (number of connected edges; force-directed layout of largest connected component shown)

this question asks whether attendees agree that "Future virtual conferences should include a tool with functionality like Minglr (to support ad hoc, private video conversations)."

For completeness, the table shows survey responses from people who did not use Minglr, but we do not believe these responses are very informative. Since these answers are presumably based only on the short descriptions of the system given in the general sessions of the two conferences, they could be affected by the quality of the description, the charisma of the presenter, or other factors. We believe it is much more useful to look at the responses of people who actually had personal experience with using Minglr, even if that is a smaller subset.

Also, the technical problems users experienced were primarily network connectivity or browser incompatibility problems, rather
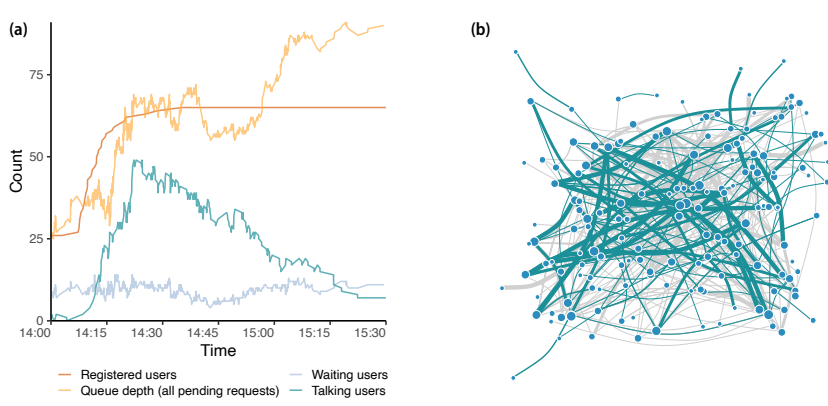

Figure 5: Minglr usage in Study 2. (a) Timeline of break period during with peak usage. (b) Communication network (grey edges are requests, green are conversations weighted by length, node size scaled by degree, force-directed layout of largest connected component shown).

than problems due to the user. It seems likely that having such problems could have biased the users' impressions of Minglr. But since our goal is to assess the usefulness of "functionality like Minglr," not the quality of our specific implementation of that functionality, we believe the results for people who did not have technical problems are more informative. So that is what we will emphasize here. However, it is possible that the opinions of people who used the system without technical difficulties are somehow not representative of what typical users of a more mature system would think.

The bottom two rows of the table show two ways of summarizing agreement with the statement. One is the total percentage of those who "Agreed" and those who "Strongly agreed." The other calculates the same total but excludes from the denominator those who said they had "No opinion."

We believe the most appropriate measure here is the one that includes "No opinion" in the denominator. While some people who said they had "no opinion" may genuinely have had no opinion (and could, therefore, be disregarded), some may also have been indicating that their opinion was neutral (i.e., somewhere between

Table 1: Desirability of including a tool like Minglr in future virtual conferences

\section{Future virtual conferences should include a tool with functionality like Minglr} (to support ad hoc, private video conversations)

\begin{tabular}{lccc|ccc}
\hline & \multicolumn{3}{c|}{ Study 1 (CI 2020) } & \multicolumn{2}{c}{ Study 2 (NFW) } \\
\cline { 2 - 7 } & $\begin{array}{c}\text { Did not } \\
\text { use Minglr } \\
(\mathrm{n}=41)\end{array}$ & $\begin{array}{c}\text { Used Minglr } \\
\text { w/o technical } \\
\text { problems } \\
(\mathrm{n}=29)\end{array}$ & $\begin{array}{c}\text { Used Minglr } \\
\text { w/o technical } \\
\text { problems } \\
(\mathrm{n}=15)\end{array}$ \\
\hline Strongly Disagree & $0.0 \%$ & $0.0 \%$ & $0.0 \%$ & - & $4.8 \%$ & $0.0 \%$ \\
Disagree & $4.9 \%$ & $6.9 \%$ & $0.0 \%$ & - & $4.8 \%$ & $0.0 \%$ \\
No Opinion & $56.1 \%$ & $13.8 \%$ & $14.3 \%$ & $100 \%$ & $23.8 \%$ & $23.1 \%$ \\
Agree & $36.6 \%$ & $24.1 \%$ & $42.9 \%$ & - & $61.9 \%$ & $76.9 \%$ \\
Strongly Agree & $2.4 \%$ & $55.2 \%$ & $42.9 \%$ & - & $4.8 \%$ & $0.0 \%$ \\
\hline \% Agree + Strongly Agree & $39.0 \%$ & $79.3 \%$ & $85.7 \%$ & - & $66.7 \%$ & $76.9 \%$ \\
\hline \% Agree + Strongly Agree & & & & & \\
(excluding No Opinion) & $88.9 \%$ & $92.0 \%$ & $\mathbf{1 0 0 \%}$ & - & $87.5 \%$ & $\mathbf{1 0 0 \%}$ \\
\hline
\end{tabular}




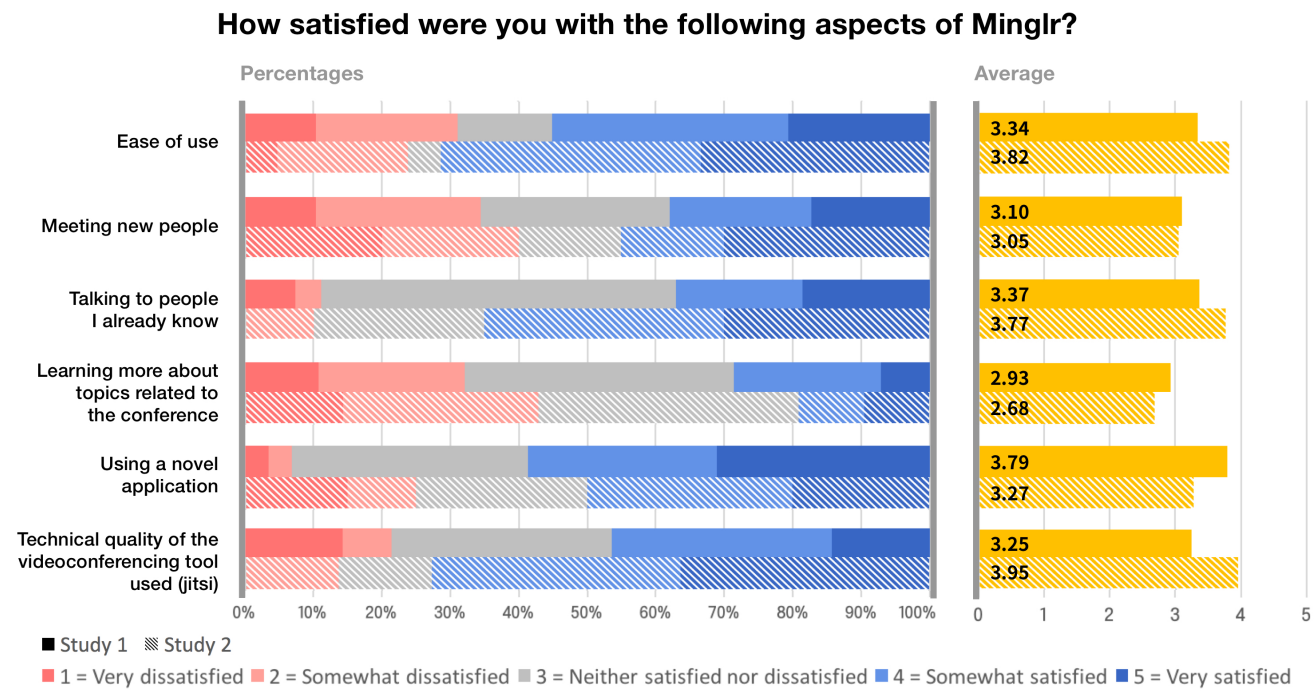

Figure 6: Satisfaction with different aspects of Minglr.

Agree and Disagree). On this (conservative) measure, the percentage agreement for people who used the system without technical problems was $86 \%$ for Study $1,77 \%$ for Study 2, and $81 \%$ averaged over the two studies.

We believe that, together, these measures of agreement from the conference attendees strongly support the claim that "functionality like Minglr" can be valuable in virtual conferences.

7.2.3 Satisfaction with various aspects of Minglr. We also measured how satisfied the attendees who used Minglr were with Minglr's various aspects (see Figure 5). For both Study 1 and Study 2, the satisfaction scores in all aspects except for learning were higher than 3 (neutral). This suggests that users were at least somewhat satisfied with all aspects of the system except its ability to help them learn more about topics related to the conference. One potential explanation for why the satisfaction with learning is low could be because, according to the interview results, people talked primarily about non-technical topics during their Minglr conversations.

For Study 1, the satisfaction score was high for "using a novel collective intelligence application" (3.79). For Study 2, the score was high for "talking to people I already know" (3.77), "ease of use" (3.82), and "technical quality of the videoconferencing tool used" (3.95).

In both studies, however, the scores for "meeting new people" were among the lowest. This suggests that many people tended to talk to people they already knew, and that newcomers who do not have a lot of acquaintances may have had difficulty when socializing with others in Minglr. The difficulties they had are elaborated below in Section 7.3.2.

\subsection{Qualitative Results}

We illustrate the qualitative results using the data from Study 1. Our analysis revealed three primary themes that were consistent across the results from the survey and interviews.
7.3.1 Ease of having private videoconferences. The results indicate that Minglr provided a very easy way for conference participants to have private videoconferences. Three out of nineteen survey respondents mentioned the ease of use as a strength of Minglr: "I liked the ease of meeting new people." Also, three out of five interviewees said Minglr made the process of interaction easier. One of them said, "it makes it easy to have sidebar conversations with people."

7.3.2 Finding conversational partners. The results also suggest that Minglr generally enhanced the process of finding potential conversational partners. In the survey, nine out of nineteen respondents mentioned that they could easily find someone to talk to using Minglr. One of them explicitly mentioned that the research interests on profiles were particularly helpful: it was sort of like a professional dating app - very fun, and a great way to meet new people, especially with keywords." In the interviews, one interviewee pointed out the usefulness of the search feature.

Still, some of the shy or novice people expressed their remaining difficulty in mingling. Three out of five interviewees reported that they had difficulty in mingling with others since they were shy or new to the community. One of them was a student preparing for graduate studies and said she felt uneasy when approaching the senior researchers. Another was an employee at a research institute who didn't know anyone else at the conference and who only got matched with people who approached him first. The last interviewee was a professor who said she was new to the collective intelligence community, and she knew only three other attendees: "But I didn't really know a lot of people there. I think if I had known a lot of people there, it would have been a very different experience."

These results suggest that although Minglr was generally successful in supporting the process of finding potential conversational partners, there is a need for additional support especially for those who are shy or new to the community.

7.3.3 Efficiency of matching people. Our results indicate that Minglr provided an efficient way to match people. In other words, 
the time waiting for the next conversation partner could be minimized with the help of mechanisms provided by Minglr. One survey respondent said in his answer that Minglr was a "very convenient tool to meet people in a short period of time." Furthermore, four out of five interviewees mentioned the efficiency as the major reason why they liked the Minglr system. One of them said, "It removes the inefficiency of scheduling a meeting one by one."

\section{DISCUSSION}

We believe the most important results of our work reported here are that relatively simple software functionality can enable ad hoc, private video conversations and that this functionality can be surprisingly useful in virtual conferences. For instance, $81 \%$ of users who used the system successfully at two separate conferences agreed that functionality like that in Minglr should be included in future virtual conferences.

We also expect that some version of the basic functionality of Minglr will be useful in many other situations, too, not just academic conferences, but also business meetings, remote work groups, classes, parties, and many other professional and social events. So we expect that some version of this functionality will eventually be implemented in most major videoconferencing systems.

Limitations of Minglr. We believe that Minglr is only scratching the surface of how to support ad hoc conversations, and we hope it helps open the door to further research on this topic. Our qualitative results, for instance, suggest that future online mingling tools should facilitate more useful connections among people who do not know each other. For example, an ice-breaking feature that matches people based on their interests could reduce the pressure on shy or new people to approach others on their own. And an introduction feature could help senior members of the community introduce newcomers to others. Another possibility is to have a random matching feature for people who want to maximize their chances of serendipitous encounters.

\section{CONCLUSION}

In this paper, we first showed with a survey that many people find ad hoc conversations one of the most valuable aspects of in-person conferences but one of the least valuable aspects of virtual conferences they experienced previously. Next, we presented the design of the Minglr system, a platform for supporting precisely these kinds of ad hoc conversations at virtual conferences and other meetings. Then, we described the use of this system at two virtual conferences and showed that attendees found the system both usable and highly useful. We also found that the Minglr system was efficient in matching attendees, and we suggested design considerations for future online mingling tools.

In summary, we believe that functionality like that provided by Minglr is likely to be widely implemented in many videoconferencing systems and to increase the feasibility and desirability of many kinds of remote work and socializing.

\section{REFERENCES}

[1] ACM. 2020. Upcoming Conferences. Retrieved 2020 from https://www.acm.org/ upcoming-conferences

[2] Thomas J. Allen and Gunter Henn. 2017. The organization and architecture of innovation: managing the flow of technology. Routledge, Taylor et Francis Group.
[3] Pierre-Alexandre Balland, Cristian Jara-Figueroa, Sergio G Petralia, Mathieu PA Steijn, David L Rigby, and César A Hidalgo. 2020. Complex economic activities concentrate in large cities. Nature Human Behaviour 4, 3 (2020), 248-254.

[4] Luís M. A. Bettencourt, José Lobo, Dirk Helbing, Christian Kühnert, and Geoffrey B. West. 2007. Growth, innovation, scaling, and the pace of life in cities. Proceedings of the National Academy of Sciences 104, 17 (2007), 7301-7306.

[5] Kevin J Boudreau, Tom Brady, Ina Ganguli, Patrick Gaule, Eva Guinan, Anthony Hollenberg, and Karim R Lakhani. 2017. A field experiment on search costs and the formation of scientific collaborations. Review of Economics and Statistics 99, 4 (2017), 565-576.

[6] CarbonNeutral. 2020. Microsoft Xbox: Microsoft certifies 825,000 Xbox consoles CarbonNeutral. Retrieved 2020 from https://www.carbonneutral.com/examples/ microsoft

[7] Christian Catalini. 2018. Microgeography and the Direction of Inventive Activity. Management Science 64, 9 (2018), 4348-4364.

[8] Kate Conger. 2020. Facebook Starts Planning for Permanent Remote Workers. (2020). https://www.nytimes.com/2020/05/21/technology/facebook-remotework-coronavirus.html

[9] Mihaly Csikszentmihalyi. 1996. Flow and the psychology of discovery and invention. Vol. 56. New York: Harper Collins.

[10] Donut. 2020. Donut. Retrieved 2020 from http://donut.com/

[11] Morten Esbensen, Paolo Tell, and Jakob E Bardram. 2014. SideBar: Videoconferencing system supporting social engagement. In 10th IEEE International Conference on Collaborative Computing: Networking, Applications and Worksharing. IEEE, 358-367.

[12] Santo Fortunato, Carl T. Bergstrom, Katy Börner, James A. Evans, Dirk Helbing, Staša Milojević, Alexander M. Petersen, Filippo Radicchi, Roberta Sinatra, Brian Uzzi, Alessandro Vespignani, Ludo Waltman, Dashun Wang, and Albert-László Barabási. 2018. Science of science. Science 359, 6379 (2018).

[13] Edward L. Glaeser. 2010. Agglomeration economics. The University of Chicago Press.

[14] Mark S Granovetter. 1977. The strength of weak ties. In Social networks. Elsevier, 347-367.

[15] Martine R. Haas and Morten T. Hansen. 2007. Different knowledge, different benefits: toward a productivity perspective on knowledge sharing in organizations. Strategic Management fournal 28, 11 (2007), 1133-1153.

[16] Morten T. Hansen. 1999. The Search-Transfer Problem: The Role of Weak Ties in Sharing Knowledge across Organization Subunits. Administrative Science Quarterly 44, 1 (1999), 82.

[17] James Higham and Xavier Font. 2020. Decarbonising academia: confronting our climate hypocrisy. Fournal of Sustainable Tourism 28, 1 (2020), 1-9.

[18] Milena Buchs Associate Professor in Sustainability. 2020. University sector must tackle air travel emissions. The Conversation (Jun 2020). https://theconversation. com/university-sector-must-tackle- air-travel-emissions-118929

[19] Siempre Inc. 2020. Online Town. Retrieved 2020 from https://theonline.town/ overview

[20] Lisa Jacobson, Jonas Åkerman, Matteo Giusti, and Avit K. Bhowmik. 2020. Tipping to Staying on the Ground: Internalized Knowledge of Climate Change Crucial for Transformed Air Travel Behavior. Sustainability 12, 5 (2020), 1994.

[21] Jitsi. 2019. Retrieved 2020 from https://jitsi.org/

[22] Kibum Kim, John Bolton, Audrey Girouard, Jeremy Cooperstock, and Roel Vertegaal. 2012. TeleHuman: Effects of 3d Perspective on Gaze and Pose Estimation with a Life-Size Cylindrical Telepresence Pod. In Proceedings of the SIGCHI Conference on Human Factors in Computing Systems (Austin, Texas, USA) (CHI '12). Association for Computing Machinery, New York, NY, USA, 2531-2540. https://doi.org/10.1145/2207676.2208640

[23] Jonathan Lazar, Jinjuan Heidi Feng, and Harry Hochheiser. 2017. Research methods in human-computer interaction. Morgan Kaufmann.

[24] Intros.at Ltd. 2020. Smart Event Matchmaking Software for Virtual, Hybrid and Live Events | Grip. Retrieved 2020 from https://grip.events/

[25] Joseph F. McCarthy, David W. McDonald, Suzanne Soroczak, David H. Nguyen, and Al M. Rashid. 2004. Augmenting the Social Space of an Academic Conference. In Proceedings of the 2004 ACM Conference on Computer Supported Cooperative Work (Chicago, Illinois, USA) (CSCW'04). Association for Computing Machinery, New York, NY, USA, 39-48. https://doi.org/10.1145/1031607.1031615

[26] Frank Nagle and Florenta Teodoridis. 2019. Jack of all trades and master of knowledge: The role of diversification in new distant knowledge integration. Strategic Management fournal 41, 1 (2019), 55-85.

[27] Stuart S Rosenthal and William C Strange. 2001. The determinants of agglomeration. Journal of urban economics 50, 2 (2001), 191-229.

[28] Feng Shi and James Evans. 2019. Science and Technology Advance through Surprise. (2019). arXiv:1910.09370 [cs.DL]

[29] ACM SIGCHI. 2020. CHI 2020. Retrieved 2020 from https://chi2020.acm.org/

[30] Paula E Stephan. 2012. How economics shapes science. Vol. 1. Harvard University Press Cambridge, MA.

[31] Hiroko Tabuchi. 2019. 'Worse Than Anyone Expected': Air Travel Emissions Vastly Outpace Predictions. The New York Times (Sep 2019). https://www. nytimes.com/2019/09/19/climate/air-travel-emissions.html 
[32] Zoom Technologies. 2020. qube - Zoom App Marketplace. Retrieved 2020 from https://marketplace.zoom.us/apps/CdxdZhqoSWCQ7vbLaLffWQ

[33] Zoom Technologies. 2020. Sococo - Zoom App Marketplace. Retrieved 2020 from https://marketplace.zoom.us/apps/0F3nAYAeQoGtt39dlpFPww

[34] B. Uzzi, S. Mukherjee, M. Stringer, and B. Jones. 2013. Atypical Combinations and Scientific Impact. Science 342, 6157 (2013), 468-472.

[35] Roel Vertegaal, Ivo Weevers, Changuk Sohn, and Chris Cheung. 2003. GAZE-2 Conveying Eye Contact in Group Video Conferencing Using Eye-Controlled Camera Direction. In Proceedings of the SIGCHI Conference on Human Factors in Computing Systems (Ft. Lauderdale, Florida, USA) (CHI '03). Association for Computing Machinery, New York, NY, USA, 521-528. https://doi.org/10.1145/ 642611.642702

[36] Andrew Winston. 2019. Should Businesses Stop Flying to Fight Climate Change? MIT Sloan Management Review (Nov 2019). https://sloanreview.mit.edu/article/ should-businesses-stop-flying-to-fight-climate-change/

[37] Andrew Winston. 2020. Is the COVID-19 Outbreak a Black Swan or the New Normal? MIT Sloan Management Review (Mar 2020). https://sloanreview.mit. edu/article/is-the-covid-19-outbreak-a-black-swan-or-the-new-normal/ 